\title{
Selective retina therapy (SRT) for macular serous retinal detachment associated with tilted disc syndrome
}

Kumiko Hirayama, Manabu Yamamoto, Takeya Kohno, Akika

Kyo, Dirk Theisen-Kunde, Ralf Brinkmann, Yoko Miura \&

Shigeru Honda

\begin{tabular}{|c|l|}
\hline Citation & Graefe's Archive for Clinical and Experimental Ophthalmology. 259(2); 387-393 \\
\hline Published & 2020-09-22 \\
\hline Issue Date & 2021-02 \\
\hline Type & Journal Article \\
\hline Textversion & Author \\
\hline Rights & $\begin{array}{l}\text { This is a post-peer-review, pre-copyedit version of an article published in Graefe's } \\
\text { Archive for Clinical and Experimental Ophthalmology. The final authenticated version } \\
\text { is available online at: https://doi.org/10.1007/s00417-020-04931-1. } \\
\text { See Springer Nature terms of reuse. } \\
\text { https://www.springernature.com/gp/open-research/policies/accepted-manuscript-terms }\end{array}$ \\
\hline DOI & \begin{tabular}{l}
$10.1007 /$ s00417-020-04931-1 \\
\hline
\end{tabular}
\end{tabular}

\author{
Self-Archiving by Author(s) \\ Placed on: Osaka City University Repository
}

Hirayama, K., Yamamoto, M., Kohno, T. et al. Selective retina therapy (SRT) for macular serous retinal detachment associated with tilted disc syndrome. Graefe's Archive for Clinical and Experimental Ophthalmology. 259, 387-393 (2021). https://doi.org/10.1007/s00417-020-04931-1 


\section{$\underline{\text { Title }}$}

Selective retina therapy (SRT) for macular serous retinal detachment associated with tilted disc syndrome.

\section{Authors}

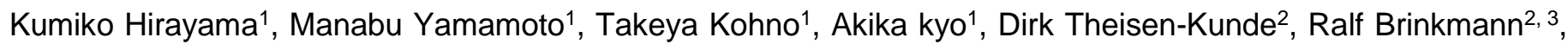
Yoko Miura $^{2-4}$, Shigeru Honda ${ }^{1}$

\section{Affiliation}

1: Department of Ophthalmology and Visual Science, Osaka City University Graduate School of Medicine, Osaka, Japan

2: Medical Laser Center Lübeck, Lübeck, Germany

3. Institute of Biomedical Optics, University of Lübeck, Lübeck, Germany

4: Department of Ophthalmology, University Medical Center Schleswig-Holstein, Campus Lübeck, Lübeck, Germany

\section{Corresponding author}

Manabu Yamamoto, M.D., Ph.D., Department of Ophthalmology and Visual Sciences, Osaka City University Graduate School of Medicine, 1-4-3 Asahi-machi, Abeno-ku, 545-8585, Osaka, Japan; Phone: +81-66645-3867; FAX: +81-6634-3873; e-mail: manabun@msic.med.osaka-cu.ac.jp

\section{Acknowledgements}

Financial Support: none.

\section{Key messages}

This manuscript presents that Selective Retina Therapy (SRT) may promote absorption of subretinal fluid in macular serous retinal detachment (MSRD) associated with tilted disc syndrome (TDS). Resolution of MSRD was observed in 6 eyes (55\%) of 11 patients who underwent SRT. Visual acuity was maintained during the follow-up period, which was averagely for 24.4 months. 


\section{Abstract}

Purpose: Tilted disc syndrome (TDS) may be associated with a macular serous retinal detachment (MSRD). However, ideal therapy for this complication is still unestablished yet to date. The purpose of this study is to investigate the effect of selective retina therapy (SRT) for macular MSRD associated with TDS.

Methods: This retrospective study included 11 eyes of 10 patients ( 1 male and 9 females), who were treated with SRT for MSRD associated with TDS, and observed at least 12 months after treatment. The mean age was 56 years old (range 44-66). An SRT laser (527 nm, $1.7 \mu \mathrm{s}, 100 \mathrm{~Hz}$; Medical Laser Center Lübeck, Germany) was used for treatment. The changes of best corrected visual acuity (BCVA), central macular thickness (CMT) and central choroidal thickness (CCT) were examined. Subfoveal curve height $(\mathrm{SFCH})$ was calculated at baseline.

Results: The mean follow-up period was 24.4 months (range 12-48 months). The mean BCVA (logMAR), CMT and CCT changed from $0.03 \pm 0.10,324 \pm 82 \mu \mathrm{m}$ and $194 \pm 68 \mu \mathrm{m}$ preoperatively to $0.07 \pm 0.17,274 \pm 94 \mu \mathrm{m}$ and $188 \pm 65$ $\mu \mathrm{m}$ at final follow-up, respectively, with significant difference on CMT (BCVA: $p=0.44$, CMT: $p<0.05, \mathrm{CCT}: p=0.21$ ). The MSRD disappeared in 6 eyes (55\%) and the average number of SRT irradiations until resolution of MSRD was 2.6 times (range 1-5 times). There was no significant association between SFCH and resolution of MSRD ( $p=0.19)$.

Conclusions: SRT may promote absorption of MSRD and maintenance of BCVA for TDS. Randomized and prospective clinical studies are needed to evaluate the effectiveness of SRT for MSRD associated with TDS.

\section{Keywords}

- laser therapy

- retinal pigment epithelium

- retinal diseases

- subretinal fluid 


\section{Introduction}

Tilted disc syndrome (TDS) is one of the congenital morphological abnormalities of the optic nerve head and often accompanied with posterior staphyloma with various macular diseases such as choroidal neovascularization, polypoidal choroidal vasculopathy, retinal pigment epithelial abnormalities and macular serous retinal detachment (MSRD) [1, 2]. As the treatment for MSRD associated with TDS, local photocoagulation, photodynamic therapy, intravitreal injection of anti-vascular endothelial growth factor (VEGF) agents has been reported; however, the effects of such treatments were insufficient [3-7].

Selective retina therapy (SRT) was developed as a laser capable for treating only the retinal pigment epithelium (RPE) without damaging neural retina and choroid [8-10]. The principle is not thermal coagulation like a conventional laser therapy, but a photomechanical disruption of RPE cells by intracellular microbubble formation after microsecond laser exposure [11]. Previous studies have been reported that SRT was effective in the central serous chorioretinopathy (CSC) and diabetic macula edema (DME) [10,12-14].

We also reported that SRT may reduce subretinal fluid in CSC without decreasing local retinal sensitivity, which confirmed its safety for the retinal nerves [12]. However, no study has been reported to date to evaluate the usefulness of SRT in the treatment of TDS. The aim of this study was thus to investigate the safety and efficacy of SRT for MSRD associated with TDS.

\section{Methods}

\section{Subjects}

This retrospective study was approved by the ethics committee of our hospital, carried out on the basis of the Declaration of Helsinki, and registered with University hospital Medical Information Network (UMIN) (No. 000010471). Written informed consent was obtained from all patients prior to enrolment. This study investigated 11 eyes in 10 TDS patients with MSRD ( 1 male and 9 females), who performed SRT in the Department of Ophthalmology at Osaka City University Hospital between March 2013 and March 2016 and were followed-up for at least 12 months. The mean age was 56 years (range, 44-66 years). The left part of Table 1 shows baseline characteristics of the patients. Two cases had a history of anti-VEGF therapy.

\section{Inclusion and exclusion criteria}

The inclusion criterion for this study was a presence of MSRD with leakage points on fluorescein angiography (FA) associated with TDS, which causes visual impairment as central scotoma, metamorphopsia, and loss of visual acuity.

Ophthalmological exclusion criteria were:

1) loss of sight in one eye;

2) optic media that is insufficiently transparent to acquire fundus images or obtain other imaging findings from the eye to be treated;

3) presence of inflammatory intraocular disorders, including infectious diseases;

4) intraocular surgery or laser treatment within 6 months;

5) intravitreal injection within the last 3 months;

6) presence of intraocular neovascularization including retina and choroid;

7) presence of comorbidity reducing visual acuity of the eye to be treated or that may require medical or surgical treatment during the study period; 
8) signs of infectious blepharitis, keratitis, scleritis, or conjunctivitis in the eye to be treated, or currently undergoing treatment for serious systemic infectious disease.

Systemic exclusion criteria were:

1) systemic inflammatory disease;

2) haemorrhagic diathesis, or other condition with current use of anticoagulant therapy judged to entail a high risk of serious haemorrhage during treatment;

3) current or possible pregnancy;

4) untreated or poorly controlled hypertension, diabetes which might affect the outcome of SRT;

5) a systemic condition judged as rendering it impossible to attend hospital for continued treatment.

\section{SRT method}

The core of the SRT laser was a Q-switched frequency-doubled neodymium-doped yttrium lithium fluoride (Nd:YLF) laser, frequency doubled to a wavelength of $527 \mathrm{~nm}$ (Medical Laser Center Lübeck, Lübeck, Germany). In a single irradiation, a short $1.7 \mu$ s laser pulse is repeated 30 times at a repetition rate of $100 \mathrm{~Hz}$. The laser beam was adjusted so that the irradiation diameter on the retina can be approximately $200 \mu \mathrm{m}$ under the use of a 1.05x magnification Mainster central field contact lens.

Since the induced thermomechanical cell damage is not ophthalmoscopically visible during SRT, the subsequent RPE cell disruption was inferred from ultrasonic waves emitted by the microbubble formation. These ultrasonic waves were measured with a transducer embedded in the contact lens [15]. This signal was processed by a software algorithm to compute an optoacoustic value (OA value) as reported earlier by Yasui et al [13], which serves as an indicator for microbubble formation and thus cell disruption.

Test irradiations were conducted around vascular arcades upper or lower part of the macula in order to decide the range of treatment laser energy. The test irradiation was performed with the energy started at about $60 \mu \mathrm{J}$, followed by increases with $20-\mu \mathrm{J}$ increments, until the $O A$ threshold value indicating microbubble formation has passed. After test irradiations, FA was performed, in order to confirm the laser pulse energy that causes the fluorescein leakage, indicating RPE junctional disruption. The lowest energy causing dye leakage was chosen as the one for the initiation of main treatment. The laser irradiation for the main treatment was applied at and around the leakage points detected on FA considered responsible for the subretinal fluid accumulation. If the OA value was below threshold during treatment, the treatment energy was adjusted such that the OA value exceeded it. Following the main treatment, FA was performed again, and if the increase of fluorescein leaks from the treated region was confirmed, the treatment was concluded.

The necessity of the re-treatment was judged every three months after treatment. In case the leakage on FA and MSRD was remained, SRT was applied to the leakage and the MSRD site in the re-treatment.

\section{Outcome measures}

All patients had a complete clinical ophthalmic examination including measurement of the best-correlated visual acuity (BCVA), slit-lamp examination, funduscopy, spectral domain optical coherence tomography (SD-OCT, SPECTRALIS ${ }^{\circledR}$, Heidelberg Engineering, Heidelberg, Germany), color fundus photography, fundus autofluorescence, FA, and Indocyanine green angiography (SPECTRALIS ${ }^{\circledR}$ ) before and every 3 months after treatment.

BCVA, central macular thickness (CMT) and central choroidal thickness (CCT) were compared between 
pre-treatment and the final visit. The decimal BCVA was converted to the logarithm of the minimal angle resolution (logMAR) units. With regard to BCVA, changes in logMAR $>0.2$ were considered significant. A change in CMT and CCT > 15\% compared with the baseline was regarded as significant as previously described [13]. The resolution rate of MSRD within the follow-up period was also examined. The Built-in software with SPECTRALIS ${ }^{\circledR}$ was used for measuring CMT, CCT and subfoveal curve height (SFCH) of the staphyloma. SFCH was calculated according to the report of Tan et al. [16]. The reference line with a length of $4000 \mu \mathrm{m}$ was drawn along the RPE centered on the fovea and the shortest vertical distance between the foveal center and reference line was measured as SFCH (Fig. 1).

The occurrence of serious adverse events such as severe visual loss, retinal hemorrhage, intraocular inflammation was examined within this period.

\section{Statistical analysis}

The changes in BCVA, CMT and CCT were evaluated using Wilcoxon's signed-rank test for significance. Associations with the resolution of MSRD and SFCH were tested using the Mann-Whitney $U$ test. The statistical software used was IBM ${ }^{\circledR}$ SPSS $^{\circledR}$ Statistics 24.0 (IBM Japan, Ltd., Tokyo, Japan), and $p<0.05$ was regarded as significant.

\section{$\underline{\text { Results }}$}

The clinical overview with pre-(baseline) and post-operative (final follow-up) BCVA, CMT and CCT, follow-up duration, total number of SRT sessions and resolution of SRF are shown in Table 1. The mean follow-up duration was 24.4 months (range 12 - 49 months). The mean number of SRT sessions was 4.2 times (range 2-9 times). As representative cases are shown in Fig. 2 and 3.

The mean BCVA (logMAR) was $0.03 \pm 0.10$ and $0.07 \pm 0.17$ at baseline and final follow-up, respectively, with no significant difference ( $p=0.44$ ) (Fig. 4a). Individually, BCVA was unchanged in 10 eyes and had deteriorated in 1 eye .

The mean CMT was $324 \pm 82 \mu \mathrm{m}$ and $274 \pm 94 \mu \mathrm{m}$ at baseline and final follow-up, respectively, with significant difference $(p<0.05)$ (Fig. 4b). Individually, CMT decreased in 5 eyes, was unchanged in 6 eyes. Mean CCT was $188 \pm 65 \mu \mathrm{m}$ and $191 \pm 67 \mu \mathrm{m}$ at baseline and final follow-up, respectively, with no significant difference $(p=0.21)$ (Fig. 4c). Individually, CMT decreased in 1 eye, was unchanged in 9 eyes and increased in 1 eye Six out of 11 patients (55\%) showed complete resolution of MSRD during the follow-up period. The follow-up duration until resolution of MSRD ranged from 2 to 18 months (mean 9.5 months) and the number of SRT session ranged from 1 to 5 times (mean 3.2 times). The residual 5 patients showed decrease of MSRD for each SRT session but no complete resolution of MSRD was seen during this follow-up. There was no significant association between SFCH and resolution of MSRD $(p=0.19)$.

During this study, no patient developed any other retinal disorders or other events attributable to laser irradiation.

\section{Discussion}

In this study, resolution of MSRD was observed in 6 eyes (55\%) of 11 patients who underwent SRT. Ilir et al reported that 6 eyes $(60 \%)$ in 10 eyes of 10 cases treated by PDT for MSRD associated with TDS showed resolution of MSRD [3]. Battaglia Parodi et al also reported that in 12 eyes of 8 cases who treated by $810 \mathrm{~nm}$ infrared diode microsecond-pulsed laser, MSRD reduced in all cases; however just 1 eye (8.3\%) showed a 
complete resolution [4]. Lee et al reported that Anti-VEGF therapy resulted in decrease of MSRD and modest visual improvement in six cases [6]. Recently, Kubota et al reported that visual prognosis was not influenced by the treatments [7]. They also reported the visual prognosis was better when MSRD resolved. The number of cases were small in all reports and the follow-up period varied. However, we considered that SRT is not inferior in effectiveness for treating MSRD associated with TDS compared with other methods. Visual outcome may improve if SRT can make a contribution to reduce MSRD.

There have been various reports describing complications associated with TDS, and MSRD is a relatively common complication which may affect visual function [17-22]. The pathological mechanism of TDS-associated MSRD has not been well disclosed yet, and it is assumed that various factors may be involved. For example, TDS often shows a point leakage in FA as seen in central serous chorioretinopathy (CSC) [18], It is hypothesized that a scleral protrusion at the edge of posterior staphyloma causes mechanical extension of the retina and choroid which alters RPE function to cause MSRD [1,21]. Persistent MSRD is known to cause irreversible visual loss. However, none of photocoagulation, photodynamic therapy, intravitreal injection of anti-VEGF drug successfully resolved MSRD associated with TDS [3-7].

Caillaux et al [19] reported that MSRD was more common in higher macular bulges, but in our cases the resolution of MSRD was not associated with macular bulges height. Although we could not find a prognostic factor related to the resolution of MSRD by SRT, a gradual reduction of MSRD was seen with repeated SRT even in the five eyes which did not show a complete resolution of MSRD. SRT is a laser technique that can treat only RPE by raising the temperature around melanosomes until microvaporization takes place without affecting surrounding tissues. It is believed that SRT disrupts the irradiated RPE and allows surrounding RPE to migrate and proliferate over the area of eliminated RPE, which might normalize the retinal metabolism to become healthier. Even in the cases showing MSRD resolved, complete absorption of MSRD required an average of 11 months from the last SRT, hence it may take time to normalize RPE function by SRT.

In the present study, SRT was administrated at the leakage sites in FA similarly to SRT for CSC as previously described [13], but half of the cases did not show complete resolution of MSRD. Unlike the CSC, the MSRD associated with TDS might be associated with a mechanical stress due to posterior staphyloma, and this might cause the functional abnormality of RPE not only at the leakage site detected by FA but also in the wider area. Thus, it would deserve consideration to irradiate a wider area with SRT to improve the pump function of RPE in the cases which remains MSRD after the initial session of SRT.

The main limitations of this study are the small sample size and lack of a control group, and some patients had a history of another treatment. On the other hand, the strength of this study is the long-term close follow-up by a single physician. A study with larger cohort would be expected to confirm the effectiveness of SRT for MSRD associated with TDS.

\section{Compliance with ethical standards}

\section{Funding}

No funding was received for this research.

\section{Conflict of Interest}


All authors certify that they have no affiliations with or involvement in any organization or entity with any financial interest (such as honoraria; educational grants; participation in speakers' bureaus; membership, employment, consultancies, stock ownership, or other equity interest; and expert testimony or patent-licensing arrangements), or non-financial interest (such as personal or professional relationships, affiliations, knowledge or beliefs) in the subject matter or materials discussed in this manuscript.

\section{Ethical approval}

All procedures performed in studies involving human participants were in accordance with the ethical standards of the institutional and/or national research committee and with the 1964 Helsinki declaration and its later amendments or comparable ethical standards. For this type of study formal consent is not required.

\section{Informed consent}

Written Informed consent was obtained from all individual participants included in the study. 


\section{$\underline{\text { References }}$}

1. Nakanishi H, Tsujikawa A, Gotoh N, et al (2008) Macula complications on the border of an inferior staphyloma associated with tilted disc syndrome. Retina 28:1493-501

2. Cohen SY, Quentel G, Guiberteau B, et al (1998) Macula serous retinal detachment caused by subretinal leakage in titled disc syndrome. Ophthalmology 105:1831-4

3. Ilir A, Neri P, Mariotti C, et al (2015) Considering photodynamic therapy as a therapeutic modality in selected cases of dome-shaped macula complicated by foveal serous retinal detachment. Ophthalmic Surg Lasers Imaging Retina 46:217-223

4. Parodi $M$, lacono $P$, Bandello $F$ (2018) Subthreshold laser treatment for serous retinal detachment in dome-shaped macula associated with pathologic myopia. Retina 38:359-363

5. Milani P, Pece A, Pierro L, et al (2010) Bevacizumab for macula serous neuroretinal detachment in tilted disk syndrome. J Ophthalmol 2010:970580

6. Lee EK, Yu HG (2019) Outcomes of Anti-vascular Endothelial Growth Factor Treatment for Foveal Serous Retinal Detachment Associated with Inferior Staphyloma. Korean J Ophthalmol 33:228-237

7. Kubota F, Suetsugu T, Kato A, et al (2019) Tilted Disc Syndrome Associated with Serous Retinal Detachment: Long-term Prognosis. A Retrospective Multicenter Survey. Am J Ophthalmol 207:313-318

8. Roider J, Brinkmann R, Wirbelauer C, et al (1999) Retinal sparing by selective retinal pigment epithelial photocoagulation. Arch Ophthalmol 117:1028-1034

9. Brinkmann R, Roider J, Birngruber R (2006) Selective retina therapy (SRT): a review on methods, techniques, preclinical and first clinical results. Bull Soc Belge Ophthalmol 302:51-69

10. Roider J, Liew SH, Klatt C, et al (2010) Selective retina therapy (SRT) for clinically significant diabetic macular edema. Graefes Arch Clin Exp Ophthalmol 248:1263-1272

11. Neumann J, Brinkmann R Cell (2006) disintegration by laser-induced transient microbubbles and its simultaneous monitoring by interferometry. J Biomed Opt 11:041112.

12. Park YG, Kim JR, Kang S, et al (2016) Safety and efficacy of selective retina therapy (SRT) for the treatment of diabetic macular edema in Korean patients. Graefes Arch Clin Exp Ophthalmol 254:1703-1713

13. Yasui A, Yamamoto M, Hirayama K, et al (2017) Retinal sensitivity after selective retina therapy (SRT) on patients with central serous chorioretinopathy. Graefes Arch Clin Exp Ophthalmol 255:243-254

14. Yamamoto M, Miura Y, Hirayama K, et al (2020) Predictive factors of outcome of selective retina therapy for diabetic macular edema. Int Ophthalmol. 40:1221-1232

15. Schuele G, Elsner H, Framme C, Roider J, Birngruber R, Brinkmann R (2005) Optoacoustic real-time dosimetry for selective retina treatment. Journal of Biomedical Optics 10:064022

16. Tan A, Yzer S, Freund B et al. (2017) Choroidal changes associated with serous macular detachment in eyes with staphyloma, dome-shaped macula or tilted disc syndrome. Retina 37:1544-1554

17. Liang IC, Shimada N, Tanaka Y, et al (2015) Comparison of clinical features in highly myopic eyes with and without a dome-shaped macula. Ophthalmology 122:1591-1600

18. Gaucher D, Erginary A, Lecleire-Collet A, et al (2008) Dome-shaped macula in eyes with myopic posterior staphyloma. Am J Ophthalmol 145:909-914

19. Caillaux V, Gaucher D, Gualino V, et al (2013) Morphologic characterization of dome-shaped macula detachment. Am J Ophthalmol 156:958-967 
20. Errera MH, Michaelides M, Keane PA, et al (2014) The extended clinical phenotype of dome-shaped macula. Graefes Arch Clin Exp Ophthalmol 252:499-508

21. Ellabban AA, Tsujikawa A, Muraoka Y, et al (2014) Dome-shaped macula configuration: longitudinal changes in the sclera and choroid by swept-source optical coherence tomography over two years. Am J Ophtalmol 158:1062-1070

22. Caillaux V, Gaucher D, Gualino V, et al (2013) Morphologic characterization of dome-shaped macula in myopic eyes with serous macular detachment. Am J Ophthalmol 156:958-967 


\section{Figure Captions}

\section{Fig.1}

The subfoveal curve height (SFCH) was measured on vertical optic coherence tomography scan by drawing an arbitrary reference line along the retinal pigment epithelium of $4000 \mu \mathrm{m}$ centered on the foveal center and measuring the shortest vertical distance between the foveal center and this reference line. CMT: central macular thickness, CCT: central choroidal thickness.

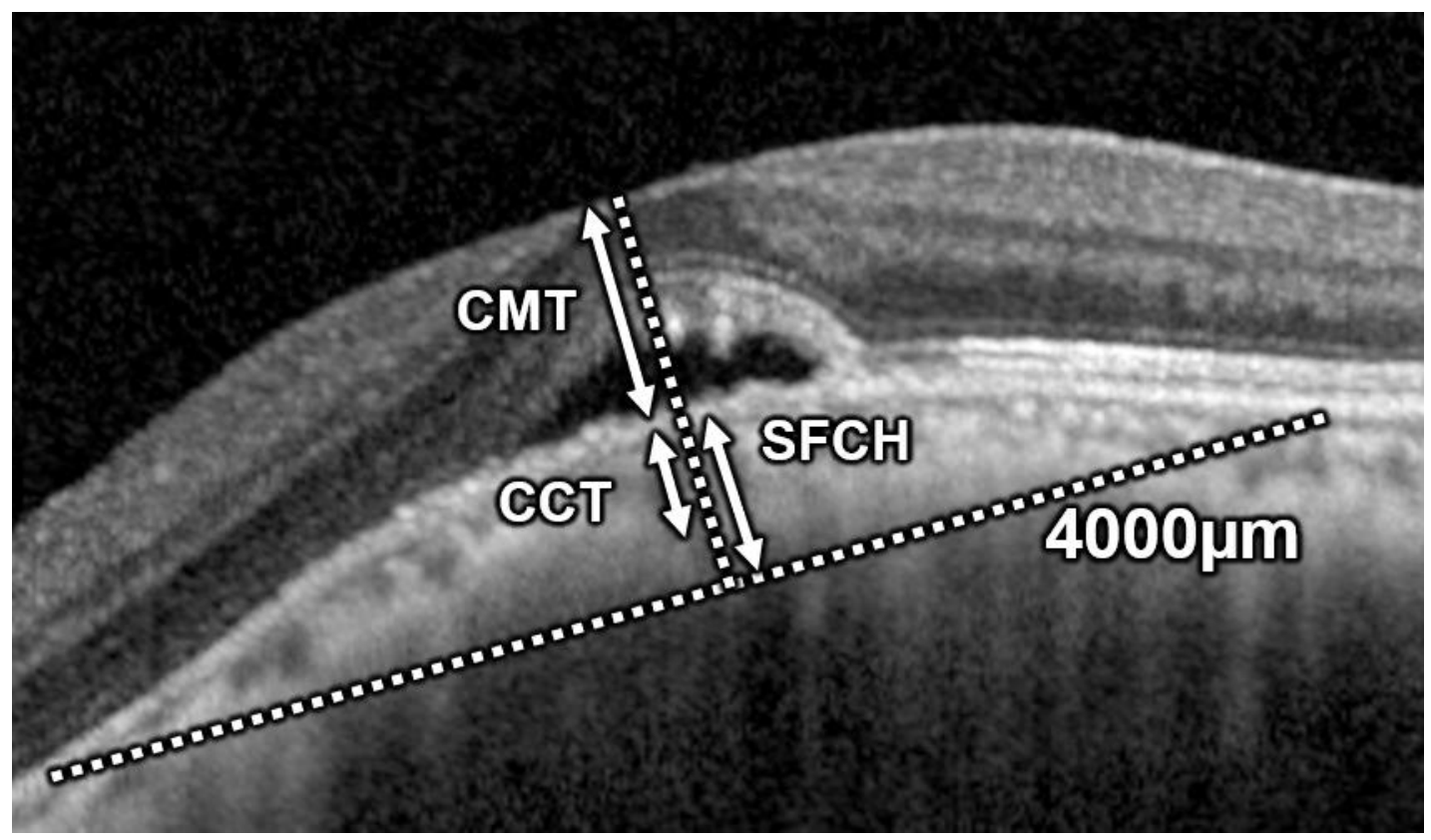




\section{Fig.2}

Clinical course of case 2 (66 years old, male, baseline BCVA is 0.00). Color fundus photograph (a) indocyanine green angiography $(b, c)$, fluorescein angiography $(d, e, f)$ and vertical line of optic coherence tomography ( $\mathrm{g}, \mathrm{h}, \mathrm{i})$ of baseline $(\mathrm{a}, \mathrm{b}, \mathrm{d}, \mathrm{g})$, 3-month follow-up $(\mathrm{e}, \mathrm{h})$ and 12-month follow-up (c, f, i) of SRT. Extent of SRT irradiation (yellow dotted line). After only one session of SRT, MSRD was resolved at 3-month follow-up and 12-month follow-up of SRT.

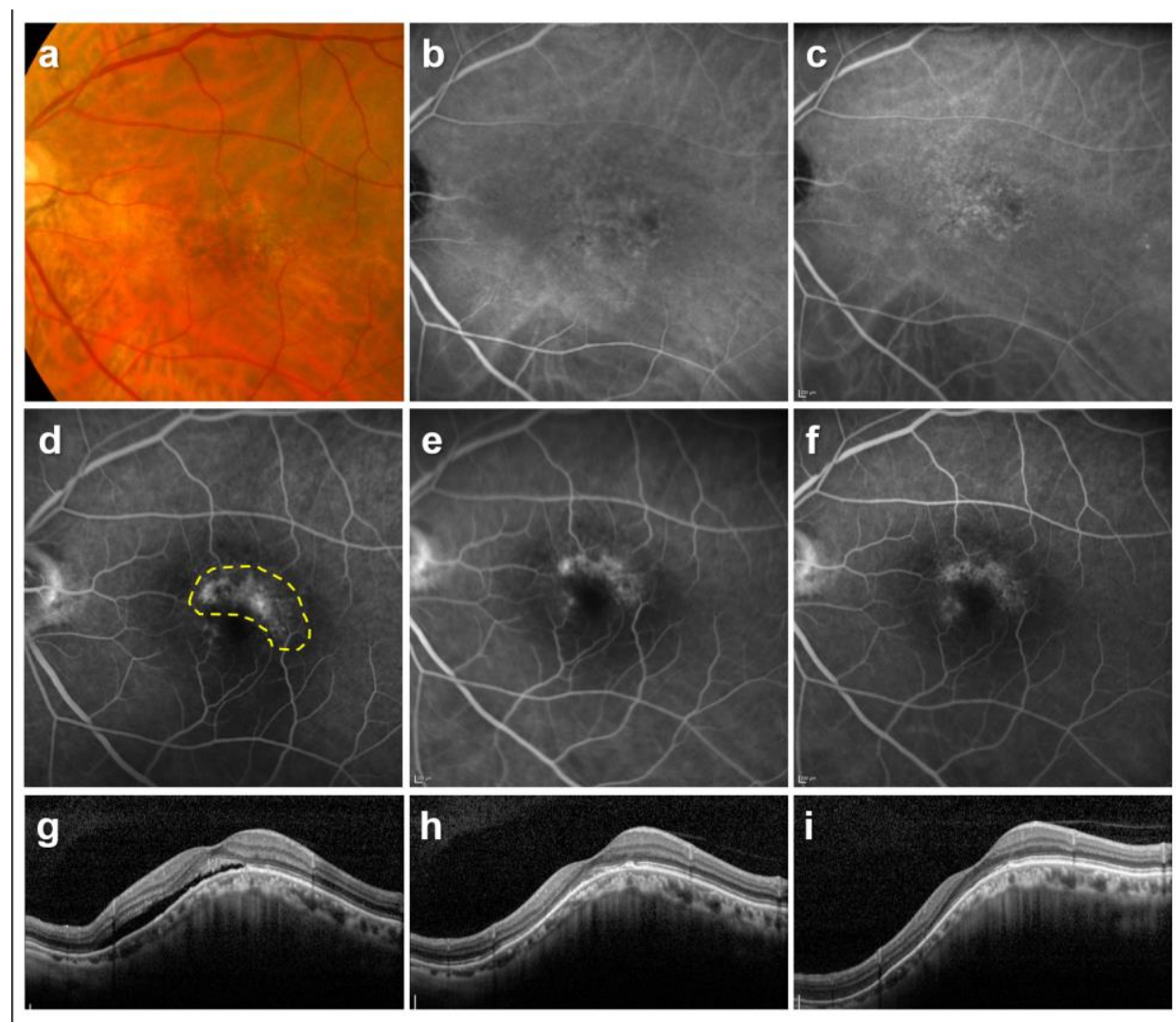




\section{Fig.3}

Clinical course of case 7 (44 years old, female, baseline BCVA is 0.05). Color fundus photograph (a), fluorescein angiography (b, $d, e, f)$ indocyanine green angiography $(c)$ and vertical line of optic coherence tomography ( $g$-l) of baseline (a, b, c, g), 3-month follow-up (d, h), 6-month follow-up (i), 9-month follow-up $(e, j), 12-m o n t h$ follow-up $(k)$, and 15-month follow-up ( $f, l)$ of SRT. Extent of SRT irradiation (yellow dotted line). SRT was performed at baseline and 9-month follow up of SRT. MSRD was resolved at 15-month follow-up of SRT.

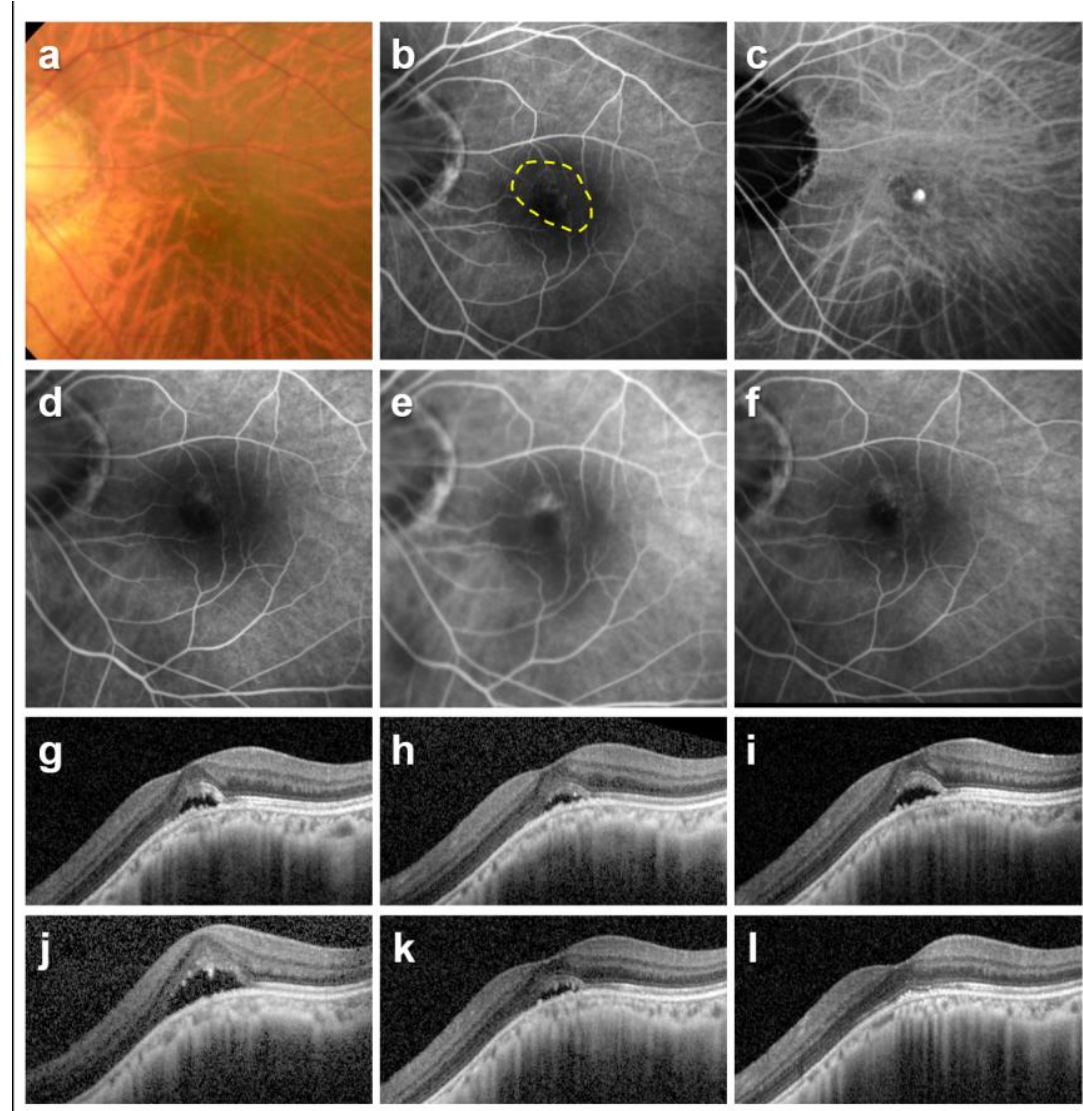


Fig.4

Changes in BCVA, CMT, and CCT before and after SRT showed by Box plots. a) The mean \pm SD of BCVA was changed from $0.03 \pm 0.10$ to $0.07 \pm 0.17$ with no significant difference $(p=0.44)$. b) The mean \pm SD of CMT was changed from $324 \pm 82 \mu \mathrm{m}$ to $274 \pm 94 \mu \mathrm{m}$ with significant difference $(p<0.05)$. $c)$ The mean \pm SD of CCT was changed from $188 \pm 65 \mu \mathrm{m}$ to $191 \pm 67 \mu \mathrm{m}$ with no significant difference $(p=0.21)$.
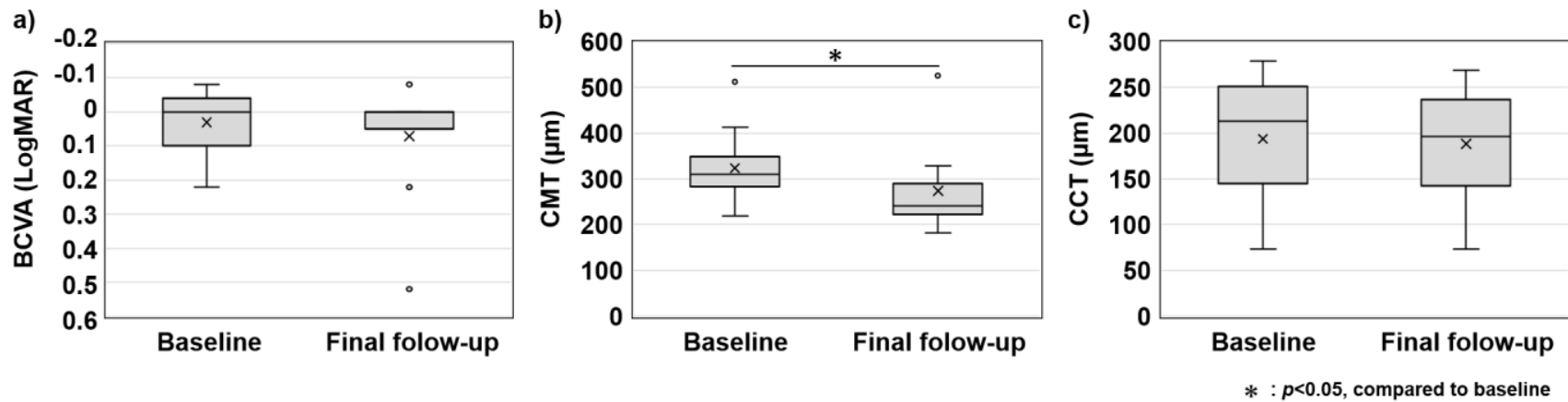
Table 1 Clinical and ocular characteristics of all patients before and after treatment

\begin{tabular}{|c|c|c|c|c|c|c|c|c|c|c|c|c|}
\hline \multirow{2}{*}{$\begin{array}{c}\text { Patients } \\
\text { /Sex } \\
\text { /Age }\end{array}$} & \multicolumn{6}{|c|}{ Baseline } & \multicolumn{6}{|c|}{ Final Follow-up } \\
\hline & $\begin{array}{l}\text { Previous } \\
\text { History }\end{array}$ & $\begin{array}{l}\text { Duration } \\
\text { (Month) }\end{array}$ & $\begin{array}{c}\text { BCVA } \\
(\log M A R)\end{array}$ & $\begin{array}{l}\text { CMT } \\
(\mu \mathrm{m})\end{array}$ & $\begin{array}{l}\text { CCT } \\
(\mu \mathrm{m})\end{array}$ & $\begin{array}{l}\text { SFCH } \\
(\mu \mathrm{m})\end{array}$ & $\begin{array}{c}\text { BCVA } \\
\text { (logMAR) }\end{array}$ & $\begin{array}{l}\text { CMT } \\
(\mu \mathrm{m})\end{array}$ & $\begin{array}{l}\text { CCT } \\
(\mu \mathrm{m})\end{array}$ & $\begin{array}{l}\text { Follow-up } \\
\text { (Month) }\end{array}$ & Tx No & $\begin{array}{c}\text { Resolution } \\
\text { (Month) }\end{array}$ \\
\hline 2/M/66 & - & 12 & 0.00 & 290 & 159 & 347 & -0.08 & 182 & 157 & 12 & 1 & Yes(3) \\
\hline 3/F/66 & $\operatorname{IVR}(6) \operatorname{IVA}(10)$ & 45 & 0.22 & 286 & 250 & 148 & 0.52 & 281 & 211 & 48 & 9 & No \\
\hline $5 / F / 49(R)$ & - & 9 & 0.00 & 310 & 279 & 364 & 0.05 & 329 & 262 & 16 & 2 & Yes(9) \\
\hline 6/F/64 & - & 5 & 0.15 & 279 & 73 & 342 & 0.22 & 232 & 73 & 25 & 3 & No \\
\hline 7/F/44 & - & 9 & 0.05 & 334 & 128 & 155 & 0.05 & 241 & 116 & 16 & 2 & Yes(15) \\
\hline 8/F/64 & - & 12 & 0.00 & 512 & 213 & 392 & 0.05 & 526 & 196 & 13 & 4 & No \\
\hline
\end{tabular}

IVR intravitreal ranibizumab, IVA intravitreal aflibercept, BCVA best corrected visual acuity, CMT central macula thickness, CCT central choroid thickness, SFCH subfoveal choroidal height, $T x$ treatment 